\title{
Acetic Acid Bacteria Supplements that Possibly Prevent Alzheimer's Disease
}

\author{
Tetsumori Yamashima* \\ Clinical Neuroscientist, Kanazawa University Graduate School of Medical Sciences, Japan
}

*Corresponding author: Tetsumori Yamashima Clinical Neuroscientist, Kanazawa University Graduate School of Medical Sciences, Kanazawa, Japan.
Received Date: July 27,2020

Published Date: August 24, 2020

\begin{abstract}
s
By covalently modifying Hsp70.1 with dual functions of recycling damaged/aged proteins and stabilizing the lysosomal membrane, 'hydroxynonenal', which derive from $\omega-6$ polyunsaturated fatty acid-rich vegetable oils (exogenous), and from membrane phospholipids and low density lipoprotein (intrinsic), may play crucial roles in the occurrence of Alzheimer's neuronal death. Aldehyde dehydrogenase is known to participate in the removal of not only ethanol-derived acetaldehyde, but also $\omega$ - 6 fatty acid-derived 'hydroxynonenal'. Here, using acetic acid bacteria as supplements with the enzyme activity of aldehyde dehydrogenase, the author perspectively reviews the most reliable preventative strategy for Alzheimer's disease.
\end{abstract}

\section{Introduction}

Acetic acid bacteria, discovered by French bacteriologist Pasteur, are essential to human life. This is because they make vinegar by breaking down the alcohol produced by fermenting sugar. For example, rice vinegar is made from rice, vinegar made from grapes, and apple vinegar and persimmon vinegar are made from fruits such as apples and persimmons. Acetic acid bacteria are essential for brewing various vinegars. Although there are other microorganisms that convert alcohol to acetic acid, the power of acetic acid bacteria is far-reaching. Acetobacter have survived for thousands of years, protecting themselves from other microorganisms by reating a highly antibacterial barrier of acetic acid around them. In addition to vinegar, acetic acid bacteria coexist with yeast and lactic acid bacteria in many naturally fermented foods such as nata de coco, fermented coconut milk, cacao, caspian sea yogurt, and kombucha (black tea mushrooms), and they all assist fermentation. In the human body, when vinegar and fermented foods are consumed, there are lactic acid bacteria and acetic acid bacteria that exist as intestinal bacteria. In the natural world too, flower nectar and tree produce sugar in large or small amounts which are converted to alcohol, and there are acetic acid bacteria in the intestines of honey bees and chimpanzees that consume them. The sugar contained in grains and sweet fruits is fermented with rice koji to make alcohol, acetic acid bacteria are added to it, and when this is left in an oven, it makes vinegar with a unique flavor. This is because the acetic acid bacteria break down alcohol into acetaldehyde, which is further broken down into acetic acid. Acetaldehyde is a carcinogen and harmful to the body, but acetic acid is completely harmless and edible, and is used to make seasonings such as mayonnaise and dressings, as well as various dishes such as sushi and pickles.

\section{Alzheimer's Disease is Endemic in Japanese People}

Alzheimer's disease is a scary disease that starts in your 40 s and destroys the brain as if strangling it with cotton for about 20 years. Initial symptoms such as forgetfulness or careless mistakes begin to appear around the age of 50, but cases diagnosed at this early stage are exceptional. Not only memory, but also attention and con- 
centration gradually decrease, and ability to make arrangements at work becomes poorer. If 10 years passes without a diagnosis, your memory problems will worsen. You'll keep on repeating the same questions and phrases when you talk to others. Thereafter, the symptoms get worse year by year, and eventually you can't say the date or where you are, and you even forget to eat. You gradually lose the ability to converse, lose your personality, can't recognize family members, and complain of hallucinations and delusions - just as if you had become another person. It is estimated that there are about 5 million Alzheimer's disease patients in both Japan and the United States. Since the population of the United States is about 2.5 times that of Japan, if there are 5 million Alzheimer's disease patients in the United States, and if the incidence rate for the population were about the same in both countries, the number of patients in Japan would be about 2 million. However, the fact that there are 5 million patients in Japan means that the incidence of the disease in Japan is 2.5 times higher than in the United States. The same is true for Western countries like Britain, France, Germany, and Italy. Compared to any other developed nation, our country has at least twice as many patients with Alzheimer's disease as a proportion of the population. So, Japanese people are highly susceptible to Alzheimer's disease.

\section{Why are Japanese People so Prone to Alzheimer's Disease?}

I think that genetic factors, environment, and food are closely related to the fact that Japanese people often get Alzheimer's disease.

\section{How are genetic factors involved?}

The people of China, Taiwan and Korea, including the Japanese, that is, rice-cultivating ethnic groups, have genetic similarities. The reason is that among these East Asians, those who cannot metabolize alcohol make up approximately $40 \%$ of the population, and their frequency is overwhelmingly higher than in other ethnic groups. I will explain how this works, later.

\section{What part does the environment play?}

Japan is a small country, with factories, airports, power plants and substations densely packed in narrow strips of land, and radio towers such as aeronautical radars, televisions, and mobile phones all over the city and suburbs. There are also many high-voltage towers, $5 \mathrm{kV}$ high-voltage electric lines stretch out vertically and horizontally, and WiFi is also popular. Most of the people use mobile phones. In other words, electromagnetic waves that cause strong oxidative stress are released into the atmosphere from these facilities, radio towers, smartphones, etc., even though they are invisible.

\section{Why does your diet matter?}

Japanese people love fried food. Tempura, fried food, cutlets, croquettes, fried fishcake, fried tofu, etc. If you add fast food or instant noodles or snacks to this list, the number of fried foods would be very numerous. In other words, Japanese people consume 13 kilograms of cooking oil a year and take excessive amounts of omega-6 linoleic acid, which is easily oxidized. Many young people consume 10 times more omega- 6 than omega- 3 fats.

\section{So how do genetics, environment and diet all come to- gether to cause Alzheimer's disease?}

Linoleic acid contained in salad oil such as soybean oil, canola oil, sunflower oil, and corn oil used to make fried food, produces a cytotoxic molecule (toxic to cells) called 'hydroxynonenal' due to the high heat used to cook fried food. Hydroxynonenal is a molecule obtained by cutting linoleic acid molecules in half, but since this molecule has an aldehyde group attached to it, it oxidizes and denatures proteins, genes and lipids, and this is why it is harmful. Lysosomes, the cells' garbage recycling centers, recycle damaged/aged proteins into amino acids, and contribute to the cell protection. In my research using Japanese macaques, hydroxynonenal oxidizes heat shock protein 70.1 (Hsp 70.1) to induce cell death by rupturing lysosomes. For example, when hydroxynonenal is injected into Japanese macaques, not only hippocampal nerve cells (neurons), but also liver and pancreas cells die. The surface of the liver is then partially whitish and degenerate enough to be visible to the naked eye. It's like a non-alcoholic steatohepatitis (NASH), which occurs in the people who drink very little alcohol but loves fried foods and high-fat foods. NASH is a serious disease, because it develops liver cirrhosis and cancer. You may not want to believe it, but this poison comes from salad oil! (Figure 1).

\section{Fried food is dangerous!}

When cooking fried foods, salad oil is heated to $180-200^{\circ} \mathrm{C}$, but heating is a strong form of oxidative stress. It therefore modifies linoleic acid, the main component of salad oil, and produces large amounts of hydroxynonenal in the oil. In addition, linoleic acid in the cell membranes of the food itself is also transformed into hydroxynonenal. For example, when shrimp is fried, the shrimp itself is full of hydroxynonenal as well as its batter coating. In the case of tonkatsu (pork cutlet), hydroxynonenal generated in the oil permeates the meat. Pork raised on solid feed such as corn and soy contains more omega- 6 fatty acids than cattle raised on pasture and grazing land. Naturally, pork cutlet also contains plenty of hydroxynonenal. In other words, if pork that originally contains some omega- 6 is fried in salad oil, it can be a dangerous food although it is meat. Although poisonous, its toxicity does not appear immediately. And it's so delicious that nobody would ever think it is a poison. This is the whole problem.

Even if you don't eat fried food, salad oil, margarine and shortening made from salad oil are often used in foods that you do often eat everyday, such as sweets, breads, cakes, donuts, cookies, ice creams, and frozen foods. This is probably because salad oil is tasty and delicious. If you look at the package ingredient table when buying sweets, cakes, sandwiches, breads, instant noodles, etc., at 
supermarkets and convenience stores, you will find, without exception, "edible vegetable oils" and "vegetable fats" marked on the package. Both "edible vegetable oils" and "vegetable fats" are salad oils themselves. And the main component of margarine and shortening is omega- 6 linoleic acid.

If you eat food that uses margarine or shortening as an ingredient even if it isn't a fried dish, linoleic acid, the main component, naturally enters the body and is taken up by cell membranes throughout the body. This is natural because the membranes surrounding the body cells are themselves made of oil. When linoleic acid taken into cell membranes is exposed to oxidative stress such as electromagnetic waves, ultraviolet rays, and exhaust gas, hydroxynonenal is generated in the body. In other words, there are two sources of hydroxynonenal: one that taken in from outside the body as food, and another which occurs naturally in the body. Now, you can understand the relationship between environment and food among the three factors of genetics, environment, and food.

\section{So what about genetics?}

The Japanese have a poorer ability to detoxify hydroxynonenal than Westerners. Surprisingly, this is inextricably linked to the fact that Japanese people cannot metabolize alcohol. The suffix "-nal" denotes a type of chemical molecule called an "-aldehyde". And, a person who is unable to decompose acet-aldehyde produced in the body from alcohol, and who cannot metabilize alcohol, also cannot detoxify hydroxynone-nal derived from salad oil. Alcohol leads to a hangover, so you notice the harm you are doing yourself. However, with food fried in salad oil, all you might notice is diarrhea or gas, so hardly anybody thinks there is a problem.

Thus, the cytotoxicity that occurs in the body due to the combination of an environment unique to Japan and the foods that Japanese people like, cannot be detoxified by the physical constitution of the Japanese people. This is the beginning of the tragedy for the Japanese. Unfortunately, most people are unaware of this.

\section{The Difference between Strong Drinkers and Weak Drinkers!}

Some people don't go red in the face even if they drink alcohol. Similarly, some people are less likely to have hangovers. But where does this difference come from? There are two enzymes, alcohol dehydrogenase (ADH) and aldehyde dehydrogenase 2 (ALDH2), which are involved in breaking down ethyl alcohol (ethanol), the main component of alcoholic drinks. ADH converts ethanol to acetaldehyde, while ALDH2 converts acetaldehyde to acetic acid, thereby detoxifying it. Japanese people have a peculiar genetic feature. Ethnically, the activity of their ADH enzyme that degrades alcohol is strong, while the activity of their ALDH2 enzyme that degrades aldehyde is weak. That said, not all people are the same, and about $40 \%$ of the Japanese have this constitution. This type of constitution makes it easier for acetaldehyde to accumulate in the body when drinking alcohol, which causes hot flushes and hangovers. People who are vulnerable to alcohol, i.e., those who have inactive

ALDH2, are more susceptible to Alzheimer's disease than those who are resistant to alcohol, i.e., those who have the active enzyme.

\section{Why is this so?}

The reason is simple. People with inactive ALDH2 can't detoxify hydroxynonenal, the causative agent of neuronal death. Hydroxynonenal destroys the heat shock protein (Hsp70.1), which transports garbage to the lysosomes, the garbage disposal plants inside cells, and makes the lysosomes strong. Therefore, if an abnormality occurs in Hsp70.1, the lysosomes explode and the neurons die. This is the "calpaincathepsin hypothesis" that I proposed in 1998. Acetaldehyde derived from alcohol disappears from the body within 24

\section{CA1 \\ liver}
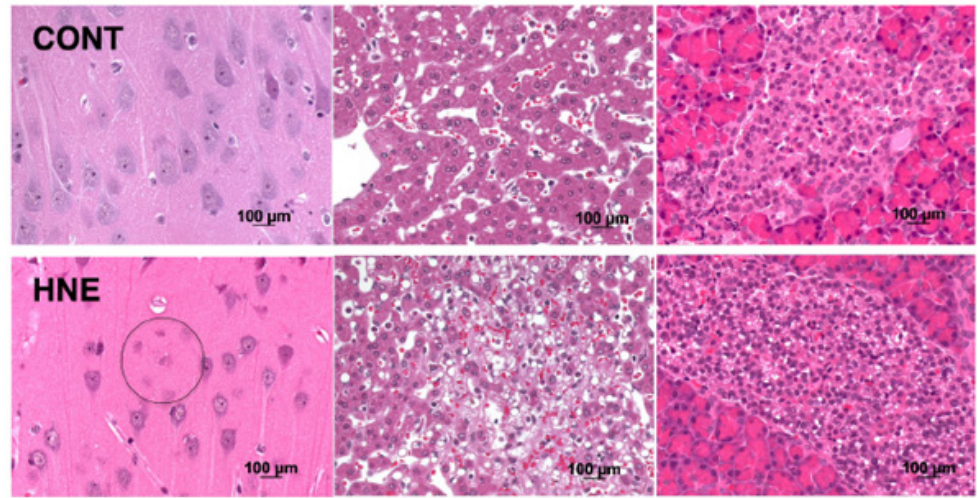

Figure 1: Injection of hydroxynonenal into Japanese monkeys kills not only the hippocampal neurons (left), but also liver (middle) and pancreas (right) cells. In the control group without injection (upper row), the cells are intact. Monkeys after hydroxynonenal (HNE) injections (lower row) show cell death in the hippocampus (left; circle) and liver (middle), and cell degeneration in the pancreas (right). Note the dramatic difference between the upper (control) and lower (hydroxynonenal injection) rows.). 


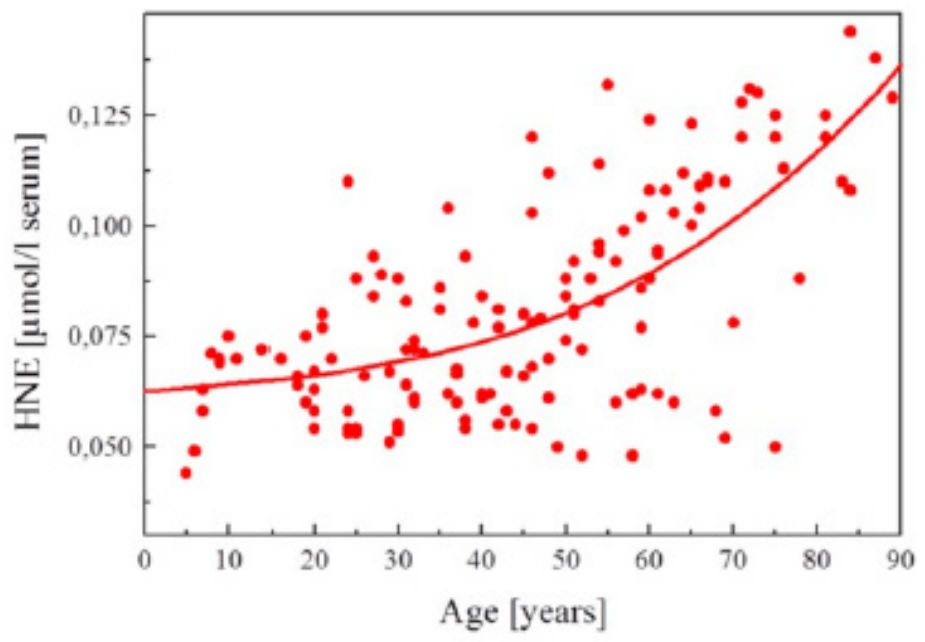

Figure 2: Hydroxynonenal accumulates in the body with age. This happens in persons who have weak resistance to alcohol, particularly those who frequently eat fried foods. Cited from 'Schaur RJ, Siems W, Bresgen N, Eckl PM (2015) 4-Hydroxy-nonenal - A bioactive lipid peroxidation product. Biomolecules 5: 2247-2337.').

hours, whether a person can take large amounts of alcohol or not. However, hydroxynonenal derived from the linoleic acid in salad oil accumulates more and more in the body (Figure 2), and as a neurotoxin accumulates in the brain although little by little. This is because hydroxynonenal adheres firmly to body debris, such as blood vessel atheromatous plaques and LDL (bad) cholesterol in the blood, and amyloid $\beta$ in the brain. And that is what causes Alzheimer's disease (Figure 2).

\section{Hydroxynonenal Concentration in the Blood In- creases with Age}

Now we see how genetics, environment, and diet are closely inter-related, and why Alzheimer's disease occurs frequently in Japanese people. There are two major pieces of evidence that hydroxynonenal is closely linked to the development of Alzheimer's disease, both clinical facts and experimental data. At present, there are no drugs that can reliably prevent Alzheimer's disease, but there is one drug that is of interest. It is a nonsteroidal anti-inflammatory drug, a so-called NSAID. Its generic name is 'flurbiprofen', and this drug is used to relieve pain in the joints associated with rheumatoid arthritis, but globally, it is known that among patients who have been taking this drug for more than a decade, there are significantly fewer cases of Alzheimer's disease. In fact, this analgesic also has the effect of activating ALDH2. If ALDH2 is activated, it means that hydroxynonenal is also easily detoxified, so that Alzheimer's disease is less likely to develop. In other words, this conversely provides evidence that hydroxynonenal causes Alzheimer's disease. Then, interesting research results using genetically-modified mice have been reported. Mice from which the gene producing ALDH2 enzyme has been deleted, model the situation of human beings that cannot tolerate alcohol. This ALDH2 gene-deficient mouse cannot make the ALDH2 enzyme, so it cannot detoxify the acetaldehyde from alcohol. Of course, hydroxynonenal derived from salad oil cannot be broken down either. The food that the mice consume every day includes soybeans and corn. When linoleic acid, which is a component of the food, is taken into their body, it is oxidized to generate hydroxynonenal. However, since they have no ALDH2, hydroxynonenal accumulates more and more. As a result, neurons in their brain die before age, the mice develop metabolic syndrome, and also Alzheimer's disease. A variety of Alzheimer's disease model mice have been created around the world, but none of them reproduce human diseases so well as these. In short, these mice simply show that hydroxynonenal is the causative agent of Alzheimer's disease.

\section{Acetic Acid Bacteria Decompose the Poison from Salad Oil}

Clearly, to avoid Alzheimer's disease, we should avoid consuming salad oil and fried foods as much as possible. But although this might seem easy, it is quite difficult to do in practice. Salad oil is tasty, and is a must in home

kitchens and restaurant kitchens. The situation is the same everywhere in the world. And nobody thinks that salad oil actually causes Alzheimer's disease. We just wouldn't want to believe it. In particular, Japanese people tend to cover up the truth instead of taking drastic measures to address the problem. That is why it is so dangerous. If you only ate raw vegetables such as vegetable salads and sashimi, boiled items such as oden and chikuzeni, grilled meat, grilled chicken, and skewered meat, etc., hydroxynonenal would not enter the body. However, sometimes people just want to eat tempura, katsudon, croquettes, and fried chicken. Unfortunately, these foods are full of hydroxynonenal. In addition, sandwiches, breads and sweets bought at convenience stores and markets use margarine and shortening. Boxed lunches also usually contain fried food. Fried dishes not only lower production costs when they are mass-produced, but also prevent food poisoning by heat steriliza- 


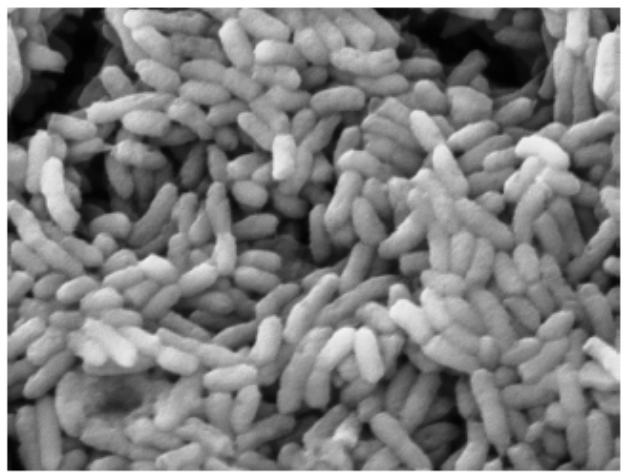

Figure 3: Electron microscope image of "acetic acid bacteria" contained in nigari vinegar, provided by Kewpie.

Acetic acid bacteria have been traditionally used by Japanese for more than 1000 years to make vinegar. When vegetables and fish are soaked in vinegar, they don't rot, don't give off unpleasant odors, and last longer. In past times when there were no refrigerators and ice, food was often preserved using vinegar. Vinegar is essential for making sushi, pickles, and mayonnaise. This vinegar is made by adding acetic acid bacteria to sake, and the acetic acid bacteria have both alcohol dehydrogenase (ADH) and aldehyde dehydrogenase (ALDH) in their pellicle. When acetic acid bacteria are added to alcohol, it turns to acetaldehyde, and finally to acetic acid. In other words, the making of vinegar from alcohol and the breakdown of alcohol in the body are actually the same mechanism. I found that these acetic acid bacteria, which are harmless to the human body, can not only break down alcohol-derived acetaldehyde, but also break down salad oil-derived hydroxynonenal. As I said earlier, "-aldehyde" (acetaldehyde) and "-nal" (hydroxynonenal) are essentially the same chemical grouping, (-CHO).

I took a thorough look at health foods (supplements) containing aldehyde dehydrogenase (ALDH) that were supposed to be an effective treatment for hangover, because there was no such drug in modern medicine. This is because a supplement that really worked for hangovers would be expected to break down not only acetaldehyde, but also hydroxynonenal. I finally selected two kinds of acetic acid bacteria supplements that were developed recently and have been sold in Japan for 3-4 years. I purchased these two products on the Internet and tried them out on three subjects. The subjects were three hospital staff: a young dentist who drinks 5 glasses of draft beer (medium-size glasses) at an izakaya restaurant in the first 30 minutes after he arrives there, a neurosurgeon who drinks 5 glasses (about $500 \mathrm{cc}$ ) of red wine every night, and a comedical who drinks 3 awamori of sake every night, and has an abnormal odor of aldehyde when he comes to work in the morning. As a result, after just one dose, the dentist shouted, "Oh, Doctor! if you take that supplement, you'll never have a hangover the next day!" The second subject was completely free from malaise and hangover the next morning even after drinking about 5 glasses of red wine. Finally, with the 50-year-old man who likes awamori, the aldehyde smell dropped sharply on the 3rd and 4th day of taking the supplement.
I thought that a supplement that could decompose acetaldehyde should also be able to degrade hydroxynonenal derived from salad oil. Although no official clinical data exists at this time, both supplements did indeed successfully detoxify hydroxynonenal in test tubes. This is enough evidence. Alzheimer's disease is so common in the Japanese population that it is not only a major social problem, but also has a negative impact on the national economy, so as an expert on the brain, I cannot ignore it. I was convinced that this information, that Alzheimer's disease could be prevented by an investment of only $\sim 60$ USD per month, should be shared with the whole world.

So in this article, I will introduce published information after giving the manufacturer's name and product names for two acetic acid bacteria supplements that are easily available online.

\section{Acetic Acid Bacteria Supplement Made from Mayonnaise Company}

Kewpie is a company that manufactures and sells mayonnaise, known for its cute Kewpie mascot. Mayonnaise is made by blending eggs with cooking oil and vinegar. Of these three ingredients, vinegar is an important one that determines the taste and quality of the mayonnaise. For over 50 years, Kewpie has produced its own high-grade vinegar for making mayonnaise. While observing the addition of acetic acid bacteria to alcohol, one of the researchers discovered that this bacteria were able to break it down, and decided to apply their potential. Normally, comestible vinegar is obtained by filtering "turbid vinegar" which contains acetic acid bacteria enzymes in high concentration. Since acetic acid bacteria enzymes are removed from commercially available vinegar by heating and filtration, Kewpie focused on raw "turbid vinegar", which was not subjected to filtration and heat sterilization, and so the acetic acid bacteria enzymes were not destroyed. With state-of-the-art technology, "turbid vinegar" was mass-produced and concentrated 1000 times, successfully producing acetic acid bacteria (Figure 3) which were encapsulated in soft capsules (Figure 4). In soft capsules, the acetic acid bacteria are hardened with oil and gelatin and are not exposed to the outside air. Although the acetic acid bacteria are no longer alive, their enzymic activity remains. 
Naturally, this product is completely harmless to the human body. Moreover, two soft capsules melt in the digestive tract, and as soon as the bacteria come in contact with acetaldehyde or hydroxynonenal, their enzymatic activity is revived and begins to de- compose these toxins. This makes it an ideal health food. It has been confirmed that even two years after the soft capsule was made, $50 \%$ of the enzymic activity remains, so its quality is maintained (Figure 4).

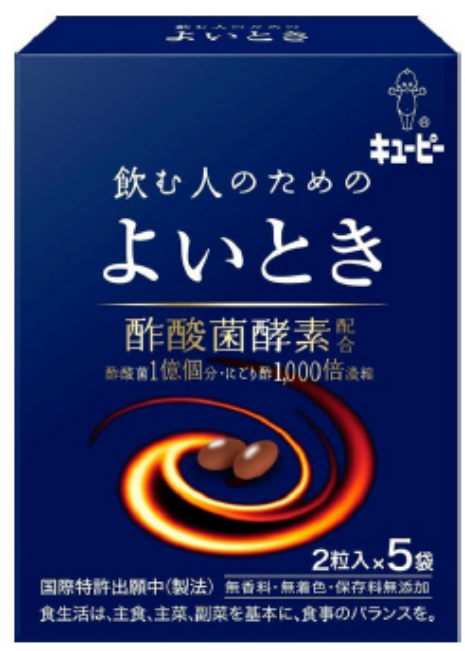

Figure 4: 'Yoitoki' from Kewpie's website.

This is the first product in their business model, which is an open system for ideas from employees, and it is truly unique. The product, "Yoitoki" (Good Times) was launched in October 2016. One pouch with two capsules

costs 200 yen ( 2 USD) including tax (Figure 4). These two capsules contain 100 million acetic acid bacteria. The total surface area of 100 million acetic acid bacteria is quite large, so sufficient enzyme comes in contact with aldehydes and hydroxynonenal in the digestive tract. If you take this before drinking, your blood alcohol level will decrease significantly. This is undoubtedly the effect of alcohol dehydrogenase (ADH) in the pellicle of acetic acid bacteria.

I myself started taking two capsules with a glass of water before an evening drink at home or a meal outside, and the drowsiness I noticed the morning after drinking a lot of wine completely disappeared. Even after drinking a lot with the meal, I felt refreshed the next morning, and free from the usual headache and nausea that I had experienced before. It was truly amazing. This effect was thanks to another enzyme in the pellicle of acetic acid bacteria, aldehyde dehydrogenase (ALDH). Since ALDH of acetic acid bacteria which corresponds to human ALDH2 is present in the pellicle, it has an effect only in the intestinal tract where bacteria exist. This is very convenient, as when fried food cooked with salad oil is digested, the enzyme will also break down the hydroxynonenal in the fried food.
As a result, the amount of hydroxynonenal that is absorbed into the blood is naturally reduced, which will definitely help prevent Alzheimer's disease.

\section{Acetic Acid Bacteria Supplement Made from Mangrove Soil Microorganisms}

Founded in Thailand, ISO SOLUTION (ISO Solution Company Ltd.), produces fermenting microorganisms and enzymes, protects the environment, applies microbial preparations in agriculture, forestry, fisheries and livestock, and develops health foods. It is a company which is only 20 years old, but their researchers are very talented. They noticed that in rivers that were heavily contaminated with organic matter from factory wastewater and domestic sewage in Bangkok, the contaminated water was purified in the mangrove colony, and only that part of the water was clarified. Hence, they realized that microorganisms from mangrove soil might be contributing to the purification of water and carried out intensive research. As a result, they found that mangrove soil microorganisms and the enzymes they produce did indeed help purify the water. Then, they turned active enzymes derived from microorganisms such as lactic acid bacteria and yeast to powder by freeze-drying, to create a multi-active enzyme complex consisting of seven bacterial enzymes called $N$-ZYME. The idea was to supplement enzymes that tend to become scarce in the body as it ages, using these microorganisms. 
When $N-Z Y M E$ is applied to soil, feed, and aquaculture ponds, the soil is purified and the water is cleaned, so that diseases of agricultural products and farmed shrimp and eels are reduced, and their quality is improved. It can also treat sludge and blue-green algae, and is now used to improve the water quality of the Konuma River. As it also contains enzymes that decompose oils and fats, it contributes to treatment of factory wastewater-containing oil, treatment of wastewater from processed food factories and restaurants that handle oil, and cleaning of kitchen areas. N-ZYME is not a chemical, but a mixture of enzymes produced by microorganisms in nature, so it is environmentally friendly and harmless to the human body. The microorganisms used in N-ZYME, which is sold as a health food, have all been identified, and have passed the microbiological safety assessment of the World Health Organization and the biotoxicity tests of the Japan Food Research Laboratories. What I noticed was that $N-Z Y M E$ contained Acetobacter aceti, or acetic acid bacteria. It can be expected that the ALDH of acetic acid bacteria would detoxify not only acetaldehyde, but also hydroxynonenal. A university lab that examined the enzyme activity of ALDH at the manufacturer's request detected 3.70 to $4.08 \mathrm{U}$ of ALDH activity per gram. Sumorit Co., located in Komatsu City, Ishikawa Prefecture, Japan commissioned a pharmaceutical company in Shizuoka Prefecture to encapsulate imported $N-Z Y M E$ in rice bran to make it into a unique health food. This is sold online under the product name "KISLip" (Figure 5). Consuming two tablets or eating fried food after consuming tablets with a full glass of water reduces abdominal heaviness and improves hangovers.

Figure 5: KISlip from Sumorit's website.

"Yoitoki " contains only the enzyme ALDH, but "KISLip" also contains lipase and amylase, which not only detoxifies hydroxynonenal but also helps digestion and absorption of fried foods (Figure 5).

\section{Hydroxynonenal is the Causative Agent of Alzheimer's Disease}

As rats continue to feed on high-fat foods, such as fast food, given as feed, POMC neurons that regulate appetite in the hypothalamus deep between the eyebrows begin to degenerate in the first week, and after 6 months, some of them die and the total number decreases. As a result, the rats lose control of their appetite, and they are not satisfied unless they eat too much, so they eat as much as they can and gain weight. These rats lose their ability to remember where they are, and both amyloid $\beta$ and Tau proteins accumulate in their brain. In other words, the same histological changes occur as in a brain with Alzheimer's disease. The weight of the human brain is about 1400 grams in adults, of which the hypothalamus weighs only 5 grams. The cell group called POMC neurons in the small hypothalamus is denatured by high concentrations of fatty acids and hydroxynonenal in a short period of only 1 to 3 months, and some of them die. This has been confirmed microscopically in the brains of laboratory animals, but abnormalities can also be detected in humans by a special examination method using MRI. At first, POMCs that regulate appetite and neurons in the hippocampus that store recent memories are damaged.

In other words, the more greasy food you eat, the more the neurons that control eating and memory decrease, so you can't control your appetite, and you won't be able to satisfy your appetite unless you eat a lot of greasy things. Then, due to this vicious cycle, body weight steadily increases, and eventually lifestyle-related diseases such as arteriosclerosis, hyperlipidemia, NASH, and type 2 diabetes rear their ugly head. At the same time, it leads to forgetfulness, lack of attention and loss of concentration, and eventually mild cognitive impairment (MCI), which is an early stage of Alzheimer's disease. Medically, Alzheimer's disease and diabetes are said to be closely related, such as the "front and back of a coin", but no researchers have given a clear answer as to why. In fact, the root cause of its onset is salad oil and hydroxynonenal from salad oil in fast foods, high-fat foods, and fried foods! Oils that enter the body are broken down into fatty acids, are and taken up by cell membranes throughout the body as an important structural component. If you consume oxidized oil from the beginning, neurons will gradually degenerate due to hydroxynoneal, causing brain dysfunctions such as forgetfulness, lack of concentration, depression, and irritability. In the pancreas, $\beta$ cells that secrete insulin degenerate which results in diabetes. In the liver, hepatocytes degenerate and die off gradually, which results in liver dysfunction. 
Even fatty acids that are easily oxidized, after they are taken up by cell membranes in the body, are oxidized when they are exposed to electromagnetic waves such as radio waves and smartphones, so that hydroxynonenal is also generated in the body.

One is hydroxynonenal that is contained in salad oil and fried foods. Another is hydroxynonenal that is generated naturally in the body due to invisible electromagnetic waves. From these two sources of hydroxynonenal, Japanese people not only increasingly suffer Alzheimer's disease, but also mental illnesses such as depression, adaptative disorders, and developmental disorders. Further, in my clinical experience, lifestyle-related diseases such as non-alcoholic steatohepatitis (NASH), type 2 diabetes, and metabolic syndrome are also increasing.

\section{Hydroxynonenal is also a Causative Agent of lifestylerelated Diseases}

Like macaques, organ damage due to hydroxynonenal is very likely to occur in Japanese people, because $95 \%$ of the genes are the same as those of Japanese macaque monkeys! Japanese people have different characteristics from Western people. It is known that in the United States and Europe, diabetes is overwhelmingly prevalent in obese people, but many Japanese people who are thin are less able to secrete insulin from the pancreas and develop diabetes. This is probably because people who are lean and have weak ALDH2 enzyme activity are unable to break down hydroxynonenal, and its accumulation degenerates $\beta$-cells in the pancreas, as shown in Figure 1. Recently, the number of young Japanese with fatty liver and NASH is increasing. And it is extraordinary that even elementary school students are developing diabetes. This is also due to the fact that adolescents eat too much junk food, B-grade gourmet, and instant noodles, increasing the level of hydroxynonenal in the blood, and damaging hepatocytes and the $\beta$ cells of the pancreas as shown in Figure 1.

It must be a Godsend from Heaven that baby neurons are born form in the hippocampus and hypothalamus even after adulthood. This means that if you eat a healthy diet and regularly use the right supplements, half of the fatty acids, which are the main components of the cell membranes of vital, newlyformed neurons, will be replaced in 3 months, and all of them in 6 months. In other words, if parts are replaced, patients with early disease can restore normal brain function and prevent lifestyle-related diseases such as Alzheimer's disease.
Can we Prevent Alzheimer's Disease and LifestyleRelated Diseases by Consuming a "Helper" before Drinking Alcohol?

Well, we need to change our whole way of thinking. Japanese people seem to believe that food made in Japan is the safest food in the world. But I do not think so. Unless the whole nation takes the risks of salad oil seriously and unless there is effective regulation, the country's future is really at stake. To overcome Alzheimer's disease, you must first overcome lifestylerelated diseases. Although it's impossible to change genetic traits and crowded populated areas, we need to urgently change our food habits. Salad oil is much scarier than cigarettes! Whereas tobacco only harms the lungs, salad oil damages all parts of the body. It is vital to change the attitudes not only of competent politicians who can verify the truth of this article, but also of Kasumi-ga-seki's elite bureaucrats, as well as that of edible oil and food company managers. And housewives, why don't you stop consuming salad oil, and do something to seriously protect the health of your families?

\section{Conclusion}

Investigating the mechanism of neuronal death in Alzheimer's disease is difficult, because only a tiny percentage of neurons is degenerating at any time point during the long prodromal period. Focusing on $\omega-6$ fatty acid- derived 'hydroxynonenal (HNE)' will help fill gaps in our knowledge that cannot be explained by the amyloid $\beta$ hypothesis. HNE carbonylates Hsp70.1, a heat shock protein with the dual functions of a chaperone protein and lysosomal stabilizer. HNE-mediated Hsp70.1 oxidization followed by calpain-mediated cleavage of carbonylated Hsp70.1, causes lysosomal neuronal death, as shown by the author's 'calpain-cathepsin hypothesis'. Aldehyde dehydrogenase (ALDH) participates in the removal of not only ethanol-derived acetaldehyde but also the similar aldehyde HNE. This review described that scavenging HNE by supplements of acetic acid bacteria containing ALDH enzymes would prevent Alzheimer's disease.

\section{Acknowledgement}

Supported by a grant from Kiban-Kenkyu (B) (19H04029) from the Japanese Ministry of Education, Culture, Sports, Science, and Technology.

\section{Conflict of Interest}

No conflict of interest. 